\title{
Preparation 8.5\%-efficient submodule using 5\%-efficient DSSCs via three-dimensional angle array and light-trapping layer
}

\author{
Yeon Hyang Sim ${ }^{1,2}$, Min Ju Yun', Seung I. Cha ${ }^{1,2}$ and Dong Yoon Lee
}

\begin{abstract}
Expanding the application area of photovoltaics to urban environments demands high efficiency under lowintensity lighting conditions, as well as omnidirectional light trapping. Dye-sensitized solar cells are of particular interest in this regard, owing to their superior electricity production in dim light; however, an improvement in dyesensitized solar cells efficiency is required for successful implementation. We developed a light-trapping layer within the photoanode of dye-sensitized solar cells and configured these cells into an angled three-dimensional (3D) array creating a submodule to improve efficiency. The light-trapping layer increases the travel distance of the light within the photoanode, thus improving electron generation by the photons of the omnidirectional incident light. The 3D angled array suppresses recombination and internal resistance losses, improving the collection efficiency by increasing the relative cell surface area with respect to the light projection area. Using the proposed configuration, we achieved a dye-sensitized solar cells submodule efficiency of $8.5 \%$ using 5\%-efficient dyesensitized solar cells with a pot-shaped light-trapping layer and a $60^{\circ}$ angled $3 \mathrm{D}$ array for the submodule. Considering that there is room for further improvement, our proposed photovoltaics configuration is expected to overcome the current limitations of dye-sensitized solar cells, thus providing promising photovoltaics modules for urban environments.
\end{abstract}

\section{Introduction}

Photovoltaic (PV) application is expected to continue its expansion into urban life ${ }^{1-3}$. This trend requires that PVs have a high efficiency under low-intensity lighting conditions $^{4}$, as well as omnidirectional light-trapping ability $^{5-7}$, to optimize electricity production from limited or fixed area/direction sources ${ }^{8-10}$. In this situation, a conventional flat panel of silicon $(\mathrm{Si})$ crystalline solar cells developed for large-scale applications provides low electricity production, owing to the weak intensity of the indirect light. Considering its limited fixed area with low electricity production, it could be disadvantageous to

\footnotetext{
Correspondence: Seung I. Cha (sicha@keri.re.kr)

${ }^{1}$ Energy Conversion Research Center, Electrical Materials Research Division, Korea Electrotechnology Research Institute, Changwon, Republic of Korea ${ }^{2}$ Department of Electro-functionality Materials Engineering, University of Science and Technology, Changwon, Republic of Korea
}

apply in urban, which has much area covered and it is hard to use large area.

Dye-sensitized solar cells (DSSCs) are highly efficient under low-light intensity or oblique incidence lighting conditions $^{11-13}$; several studies have reported $>25 \%$ efficiency in this type of environment ${ }^{14,15}$, a value much higher than that of conventional Si-based crystalline solar cells. However, DSSCs are limited in their operation in outdoor environments, owing to the low-light absorption coefficient of the photoanode for charge generation and slow diffusion of electrons in the photoanode for charge collection ${ }^{16-18}$. From controlling absorption coefficient for diffusion and generation of charge by modeling and modification of structure, it could enhance DSSC performance like electricity production as well as efficiency ${ }^{17}$. As such, the DSSCs must be modified to optimize their performance in urban environments. 
Here, we propose a new DSSC design involving omnidirectional light trapping with integration of a light-trapping layer in the photoanode of the DSSCs and arranging the modified DSSCs into an angled three-dimensional (3D) cell array. This angled 3D cell array concept we have introduced and proved its effect before $^{19}$. This DSSC submodule design is derived from efforts to overcome the limitation mechanisms of DSSC performance in urban applications with fixed projection areas. The angled 3D cell array increases the cell surface area for the given fixed projection area, effectively reducing the recombination of generated charges and overpotential at each electrochemical interface. Here, furthermore, integrating light-trapping effect to angle $3 \mathrm{D}$ cell array, we have modified DSSC performance. For the charge-generation feature, the light-trapping layer increases the light travel path within the photoanode for improved light absorption within the thin photoanode; thus, this modification effectively increases the DSSC light absorption coefficient. This concept greatly contributes its application into urban life by improving electricity production and cell efficiency.

Applying these two concepts, we achieved a DSSC submodule efficiency of $8.5 \%$ using DSSCs with a $5 \%$ photoanode area-based efficiency individually. It should be emphasized that the cells were not optimized at this stage of testing, regarding the light-trapping layer or 3D cell array. Also, it will be first trial of utilizing regularly arranged micro-sized light-trapping layer and 3D angled array to submodule with light distribution simulation within photoanode. Thus, modifications to the cell structure, the material components, and the designs of the light-trapping layer and 3D cell array are expected to further enhance efficiency and electricity production. We attribute these results to the hidden potential of DSSCs; as such, additional studies are required to optimize the DSSCs for implementation in urban environments.

\section{Experimental details}

\section{Two-dimensional ray tracing}

Two-dimensional ray tracing calculations were performed with an in-house code written with MATLab. For the calculations, the space was divided into pixels according to the object geometry.

In the simulations, each ray travels for so many unit pixels according to its position and propagating orientation. When the ray hits the interface, it undergoes both refraction and reflection according to the Fresnel equation. The refracted ray continues to propagate; however, the reflected ray is now represented as a new ray generated at the interface. Each ray continues its propagation until it reaches the end of the calculation space or until the beam energy decreases to $<0.1 \%$ of its initial energy. At the interface, reflection and refraction are given by the following:

$$
\begin{aligned}
& \vec{r}=\vec{i}-2(\vec{i} \cdot \vec{s}) \vec{s}:(\text { Reflection }) \\
& \vec{t}=\frac{n_{i}}{n_{t}} \vec{i}+\left\{\frac{n_{i}}{n_{t}} \cos \theta_{i}-\sqrt{1-\left(\frac{n_{i}}{n_{t}}\right)^{2}\left(1-\cos ^{2} \theta_{i}\right)}\right\} \vec{s}:
\end{aligned}
$$

(Refraction)

$$
\theta_{i}=-\cos ^{-1}(-\vec{i} \cdot \vec{s}):(\text { incident angle to surface })
$$

Where $\vec{r}$ and $\vec{t}$ are unit vector representing reflected and refracted ray, respectively, $\vec{i}$ is a unit vector for incident ray, and $\vec{s}$ is a unit vector for normal direction with respect to the interface. To calculate the light distribution within the DSSCs, the pixel size was fixed for $1 \mu \mathrm{m}$ and one ray per $1 \mu \mathrm{m}$ was considered.

\section{DSSC preparation}

Fluorine-doped tin oxide (FTO) glass (sheet resistance: $7 \Omega \mathrm{sq}^{-1}$, Sigma Aldrich, St. Louis, MO, USA) was used as the substrate for the photoanode and counter electrode. The FTO glass was rinsed with acetone, ethanol, and distilled and deionized water by sonication and dried with nitrogen gas. Then a blocking layer (Ti-Nanoxide BL/SP, Solaronix) was deposited onto the FTO glass via an automatic screen-printing process (AutoMax). The blocking layer was heated at $530{ }^{\circ} \mathrm{C}$ for $3 \mathrm{~h}$. $\mathrm{TiO}_{2}$ nanoparticles $20 \mathrm{~nm}$ in size (Ti-Nanoxide T/SP, Solaronix) were deposited onto the blocking layer and sintered at $500{ }^{\circ} \mathrm{C}$ for $1 \mathrm{~h}$, creating an area of $10 \mathrm{~mm} \times 10 \mathrm{~mm}$ in size. Photoanode thickness was controlled by repeating screenprinting times. Two times printing formed $7.5 \mu \mathrm{m}$ and four times printing formed $11.5 \mu \mathrm{m}$ of photoanode. $\mathrm{TiO}_{2}$ particles $500 \mathrm{~nm}$ in size (STP-500N, ENB Korea) were deposited onto the $20 \mathrm{~nm} \mathrm{TiO}_{2}$ nanoparticle layer and then sintered at $500{ }^{\circ} \mathrm{C}$ for $1 \mathrm{~h}$ in air. Then, the $\mathrm{TiO}_{2}$ deposited FTO glass was immersed in an ethanol solution (Sigma Aldrich) of $0.3 \mathrm{~mm}$ N719 dye $(0.3 \mathrm{~mm}$, Sigma Aldrich) for $20 \mathrm{~h}$ at room temperature. For counter electrode, platinum paste (Platisol T/SP, Solaronix) was deposited onto the FTO glass using the doctor blade method and heated at $480^{\circ} \mathrm{C}$ for $30 \mathrm{~min}$ in air. To make the cell, the photoanode and the counter electrode were assembled using $60 \mu \mathrm{m}$ Surlyn film (Meltonix 1170-60, Solaronix) and hot press.

The liquid electrolyte was then injected through the hole on the FTO-coated glass of counter electrode into the prepared cell. The liquid electrolyte was composed of $0.6 \mathrm{M}$ 1-butyl-3-methylimidazolium iodide, $0.03 \mathrm{M}$ iodine, $0.1 \mathrm{M}$ guanidine thiocyanate, $0.5 \mathrm{M}$ 4-tert-butylptridine, 
and $0.1 \mathrm{~m}$ lithium iodide dissolved in mixed acetonitrile and 3-methoxypropionitrile at a 2:8 volume ratio.

Four DSSCs connected in parallel were arrayed on flat and oblique $\left(30^{\circ}, 45^{\circ}\right.$, and $\left.60^{\circ}\right)$ frames to create submodules. Active area of connected four cells is $4 \mathrm{~cm}^{2}$ for flat array, $3.464 \mathrm{~cm}^{2}$ for $30^{\circ}, 2.828 \mathrm{~cm}^{2}$ for $45^{\circ}$, and $2 \mathrm{~cm}^{2}$ for $60^{\circ}$ angled array. Detailed illustration for devices' area is shown in Fig. S1.

\section{Fabrication of the light-trapping layer}

A Si wafer was utilized as a substrate in the master mold. For patterning the master mold, PR (AZ 9260, AZ Electronic Materials) was spun onto the $\mathrm{Si}$ wafer at $3500 \mathrm{rpm}$ for $30 \mathrm{~s}$ using a spin coater (Midas System Co., Ltd., Daejeon, Korea), creating a coating thickness of $7 \mu \mathrm{m}$. Then, the wafer was baked at $110^{\circ} \mathrm{C}$ for $10 \mathrm{~s}$. The PR was patterned by photolithography, exposed to ultraviolet light using a mask aligner (MA-6, Suss Microtec, Munchen, Germany), and then removed utilizing developer, tetramethylammonium hydroxide $(2.38 \%)$ to create a $200 \mu \mathrm{m}$ sized hexagonal array. Dry etching was conducted using a deep reactive ion etcher with cycling between Bosch and isotropic etching modes. Then the etched Si wafer was cleaned for the next step.

Polydimethylsiloxane (PDMS) (SR-580, Heesung STS) was coated onto the master mold using a spin coater (VSF-150MD) at $2000 \mathrm{rpm}$ for $40 \mathrm{~s}$ for uniform thickness of based film with $60 \mu \mathrm{m}$ and lens height were formed with $90 \mu \mathrm{m}$ for pot shape, $50 \mu \mathrm{m}$ for parabolic shape and $45 \mu \mathrm{m}$ for parabolic shape with rough surface lighttrapping lens (Fig. S2). Then these were baked at $70^{\circ} \mathrm{C}$ for $20 \mathrm{~min}$. The cured PDMS light-trapping layer was peeled from the master mold. PDMS light-trapping layer was rinsed with ethanol to remove the dust and then put on the surface of FTO-coated glass substrate.

The etching process was commissioned by the National Institute for Nanomaterials Technology, Pohang University Science and Technology.

\section{Characterization}

FE-SEM (Hitachi S4800, Tokyo, Japan) was performed to observe the surfaces and cross-sections of the lighttrapping layer. A solar simulator (Model Sun 2000, $1000 \mathrm{~W}$ Xe source, Abet Technologies, Milford, CT, USA) and Keithley 2400 source meter (Keithley Instruments, Cleveland, OH, USA) was used to evaluate the PV performance of the DSSCs under 1 sun AM1.5 conditions, as calibrated by a KG-3 filter and a National Renewable Energy Laboratory (NREL, Golden, CO, USA)-certified reference cell.

\section{Results and discussion}

DSSCs have a low-light absorption coefficient compared with other types of thin-film solar cells, thus requiring a thick photoanode for optimal photon absorption. However, DSSCs have a relatively slow electron diffusivity compared with single-crystal semiconductor-based solar cells, in which a thick photoanode enhances the recombination $\operatorname{rate}^{20-22}$. This disharmony between photon generation and recombination is considered as the primary issue limiting DSSC efficiency. If light is incident at an oblique angle (oblique illumination), the light path length within the photoanode increases and has the same effect as increasing the light absorption coefficient, as shown in Fig. 1a; this is the main principal used in lighttrapping applications. In the case of a DSSC, a sufficiently oblique angle can increase the light travel length within
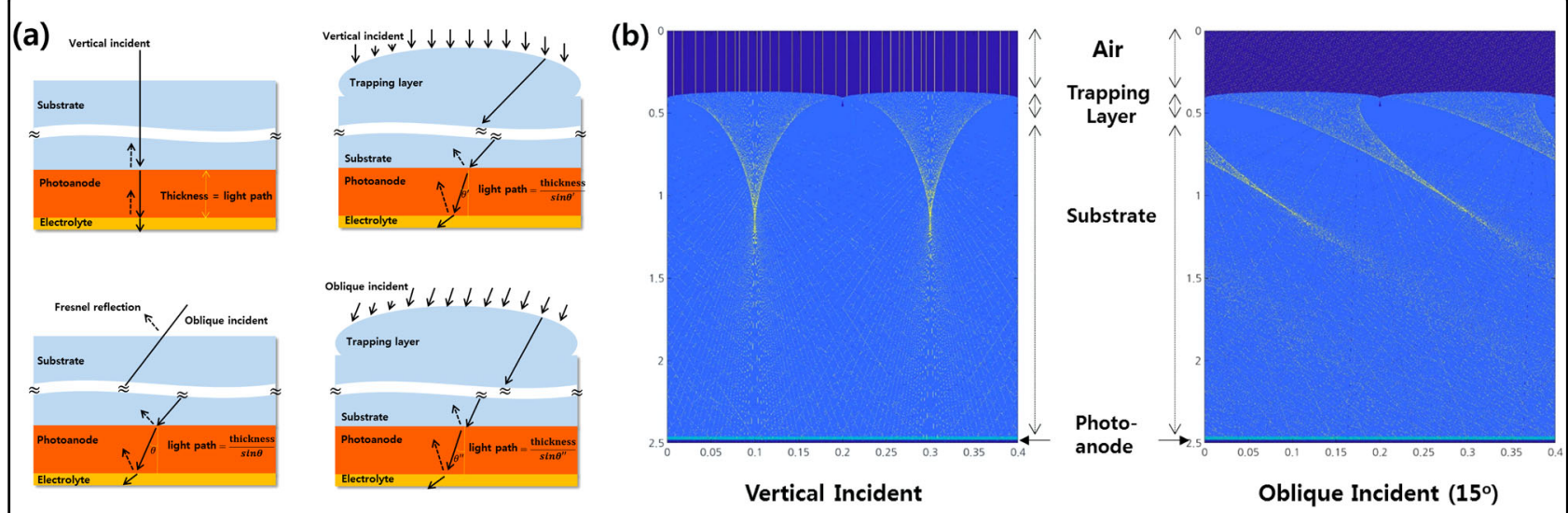

Fig. 1 a Schematic diagram showing light incident on the photoanode and the light-trapping layer. (left/right top) Light at normal incidence and (left/right bottom) oblique incidence with respect to the photoanode surface without a light-trapping layer/with a lens-shaped light-trapping layer. b Two-dimensional (2D) ray tracing simulation results showing the light propagation path within the light-trapping layer, the glass substrate, and the photoanode with a lens-shaped light-trapping layer of $50 \mu \mathrm{m}$ in height (left) at an oblique angle of $15^{\circ}$ (right). 
the photoanode. However, some portion of the light is reflected at the cell surface as Fresnel reflection ${ }^{23-26}$.

Figure 1 shows a schematic diagram of the DSSC with light incident on the photoanode. To achieve the same enhancement of oblique illumination on the light path (i.e., increase the light absorption coefficient), we introduced a light-trapping layer to the photoanode, as shown in Fig. 1a. The diagram shows the effects of light illumination at normal incidence and that of light at oblique incidence with respect to the photoanode surface, with and without the incorporation of light-trapping layers. Figure $1 \mathrm{~b}$ shows two-dimensional (2D) ray tracing simulation results of the light propagation path within the light-trapping layer, the glass substrate, and the photoanode with a lens-shaped light-trapping layer of $50 \mu \mathrm{m}$ in height and an oblique angle of $15^{\circ}$. A welldesigned light-trapping layer can accommodate all incident light directions to minimize Fresnel reflection, as proven using $2 \mathrm{D}$ ray tracing calculations. For example, if the light-trapping layer has a pot shape, the path of light at normal incidence is modified as shown in Fig. 1b, such that the light spreads after coming to a focus and falls at oblique incidence on the photoanode. The modification of the light path is sensitive to the geometry of the lighttrapping layer. The pot-shaped geometry spreads the light homogeneously onto the photoanode with little Fresnel reflection, while at the same time it delivers focused light into the photoanode at a slightly different focal length, as shown in Fig. S3.

Figure 2 shows a schematic diagram of the fabrication process of the polydimethylsiloxane (PDMS) lighttrapping layer via casting on an etch-patterned $\mathrm{Si}$ wafer. Patterns in the light-trapping film layer were formed via etching. The difference in refractive index between the PDMS and glass substrate was small; thus, photon loss from the light-trapping film was minimal ${ }^{27,28}$. The asprepared PDMS film was simply put on the surface of FTO-coated glass substrate. There Is strong adhesion between the PDMS light-trapping film and the glass so the film can be easily put on the glass without an intermediate air layer between them. Figure 2a shows photographs of the DSSCs with and without the light-trapping layer, in which the photoanode size is $1 \mathrm{~cm} \times 1 \mathrm{~cm}$. The introduction of the light-trapping layer caused a slight blurring effect; however, overall, there was little difference between the two configurations. Several geometries of the light-trapping layer were fabricated by varying the etching parameters, such as the photoresist (PR) thickness and etching time. Figure $2 \mathrm{~b}-\mathrm{d}$ shows field-emission scanning electron microscopy (FE-SEM) images of fabricated lighttrapping layers with a pot shape, a parabolic lens shape, and a parabolic lens shape with a rough surface, respectively; the insets show cross-sectional views of the lighttrapping layers. In the plane view, there is little difference among the geometries; all appear to be compact hexagonal arrays of convex parabolic lenses (i.e., conventional lenses) as shown in Fig. S3 as master mold FE-SEM images according to each shape. The close-packed array of the light-trapping layer pattern minimized reflection losses. The cross-sectional views, however, showed a clear difference among the different lens shapes (Fig. 2b-d). The light-trapping behavior, and thus the light distribution within the photoanode, depends on the geometry of the light-trapping layer. Next, 2D ray tracing simulations, in which the Beer-Lambert law was assumed, were conducted to resolve the differences among the geometries tested.

Figure 3 shows the light distribution in the lighttrapping layer, substrate, and photoanode under illumination at normal incidence, with respect to the presence and shape of the photoanode light-trapping layer. As expected, slight changes in the cross-sectional geometry created large differences in the light-trapping behavior, resulting light distribution within the photoanode, as shown in the Fig. 3a. When the focal points of the lighttrapping layer were located within the glass substrate, the light spread into the photoanode. However, the potshaped light-trapping layer exhibited a more homogenous distribution, compared with the parabolic lens shape, in which light from each lens pattern overlapped at a specific part of the photoanode. When no light-trapping layer was applied, the oblique incident light at $45^{\circ}$ created a higher light intensity near the FTO-photoanode interface, compared with that at normal incidence; however, the light intensity decreased sharply compared with that of normal incidence, as shown in Fig. 3b. The introduction of the pot-shaped light-trapping layer changed the light distribution upon illumination at normal incidence, as shown in Fig. 3c; compared with the profile shown in the absence of the light-trapping layer, the pot-shaped lighttrapping layer created a higher light intensity near the FTO-photoanode interface. Interestingly, light incident at an oblique angle concentrated the light near the interface, thus effectively increasing photon absorption in this area. In the case of the parabolic lens-shaped light-trapping layer (Fig. 3d), the overlapping rays induced lateral distribution of the light intensity within the photoanode. In addition, as shown in Fig. 3a, a considerable portion of light was reflected from the surface and other interfaces; hence, the light intensity decreased near the FTO-photoanode interface. We also examined the light distribution profile within the photoanode as a function of the distance from the FTO-photoanode interface for light at normal incidence and oblique light at $45^{\circ}$ incidence (Fig. 3e, f); the light distributions within the photoanode with different light-trapping layers were fitted to the Beer-Lambert law. Under illumination at normal incidence, the introduction of the light-trapping layer did not 


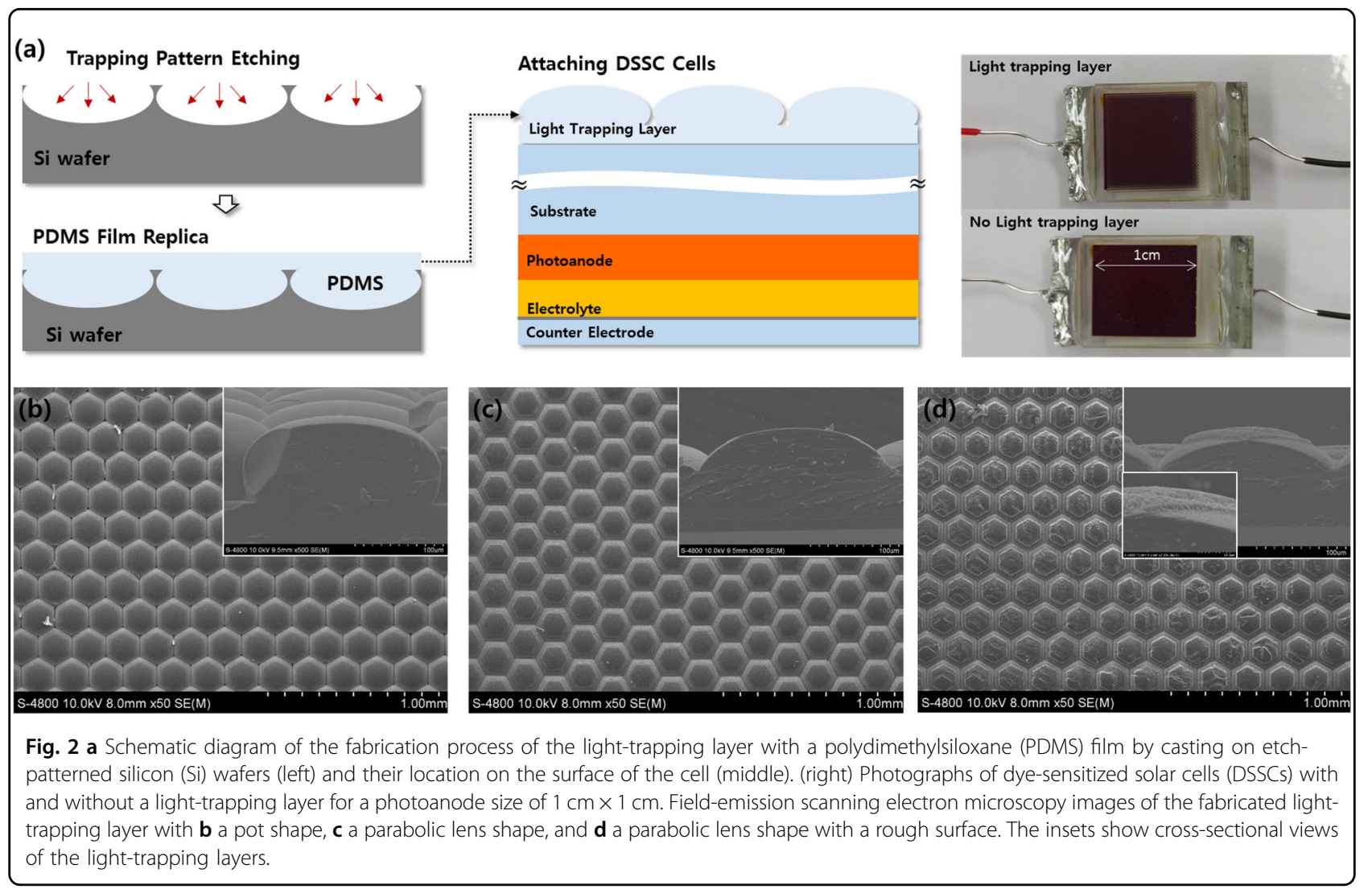

have a significant effect on the light absorption, similar to the effect observed at a $45^{\circ}$ oblique angle of incidence without the light-trapping layer. However, in the presence of a light-trapping layer, light at oblique incidence increased the light absorption coefficient by $15-20 \%$. Thus, a well-designed light-trapping layer enhances the charge generation within the photoanode by modifying the light path, i.e., increasing the light travel length (or effective light absorption coefficient). We suggest that additional investigation into the design of the lighttrapping layer is needed to further improve the effective charge generation within the DSSC photoanode, such that the light absorption coefficient of a DSSC approaches that of a direct band gap-structured thin-film solar cell.

For comparison, we examined the effects of photoanode thickness on the resulting normalized current density and conversion efficiency of the DSSCs for two thicknesses: 7.5 and $11.5 \mu \mathrm{m}$, as shown in Fig. 4. PV performance of relationship between voltage and current density according to phtoanode thickness with shape of lighttrapping and oblique angle were shown in Fig. S5 for $7.5 \mu \mathrm{m}$ thickness and Fig. S6 for $11.5 \mu \mathrm{m}$ thickness. Also PV performance parameters value were shown in Table S1, which are average one of four cells with same conditions. The modified light distribution by the light-trapping layer clearly affected the short-circuit current density $\left(J_{\mathrm{sc}}\right)$.
For a thin photoanode thickness of $7.5 \mu \mathrm{m}, J_{\mathrm{sc}}$ was strongly affected by the light distribution near the FTO-photoanode interface, as expected from the light distributions within the photoanode (Fig. 3). Specifically, the pot-shaped light-trapping layers enhanced $J_{\mathrm{sc}}$ for all incident angles, whereas the parabolic lens array had little effect. However, the energy conversion efficiency did not directly match the Jsc that affected open-circuit voltage and fill factor as well. Through light-trapping layer, current was increased, but through light distribution, internal resistance in inner DSSC was changed, which affects voltage and fill factor. Therefore, the energy conversion efficiency did not increase for the illumination at normal incidence by inserting the light-trapping layer, despite the increase in light intensity within the photoanode, i.e., increased $J_{\text {sc }}$.

When light illuminates a DSSC at oblique incidence, the enhanced charge generation can be utilized to enhance the energy conversion efficiency. The oblique angle of incidence effectively decreases the absolute value of the incident light intensity owing to the reduction in the projection area of the incident light. Thus, for the same photoanode area, the absolute value of the current decreases, which in turn reduces the internal voltage drop. As shown in Fig. 4e, the power loss owing to the internal voltage drop, i.e., internal 


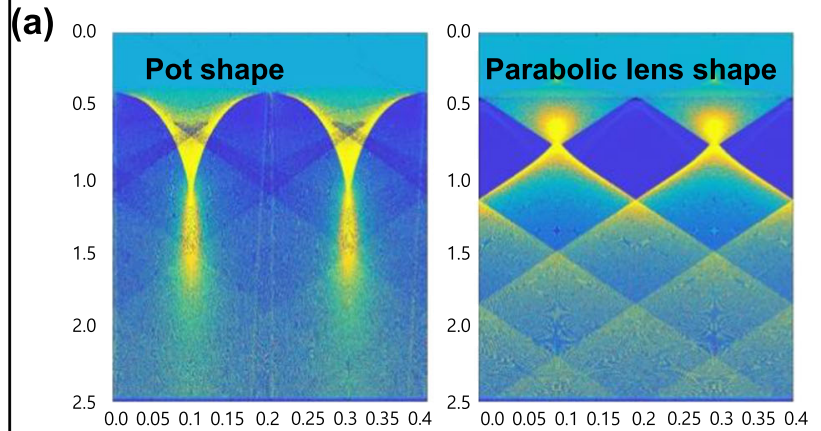

(c)
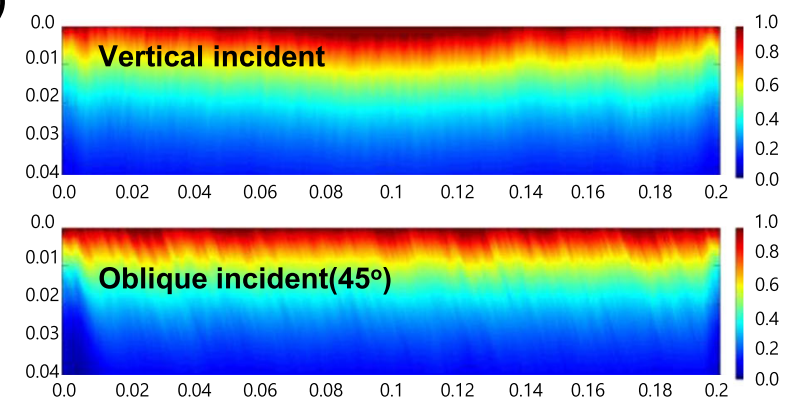

(e)

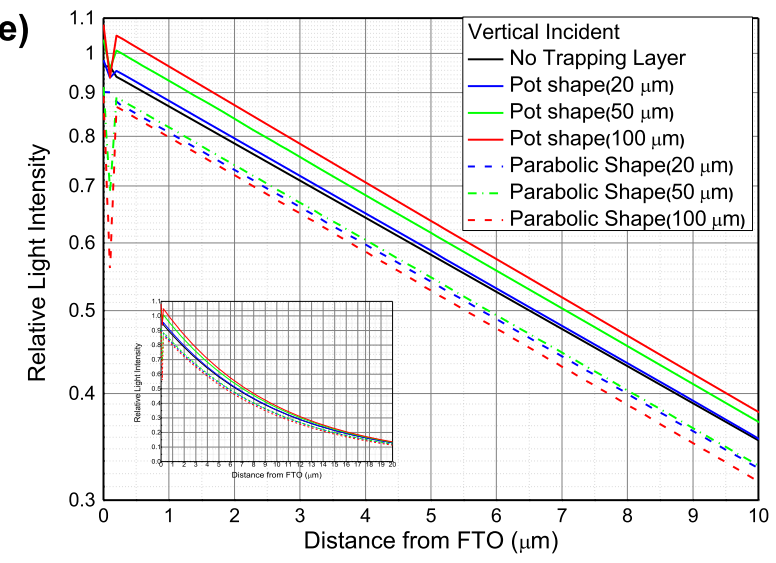

(b)
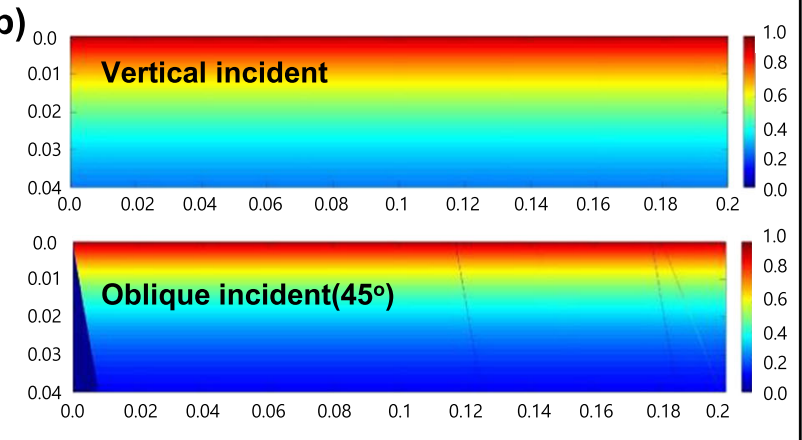

(d)
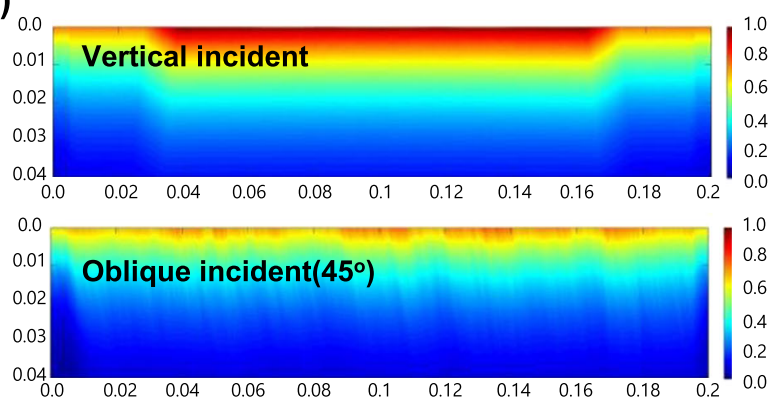

(f)

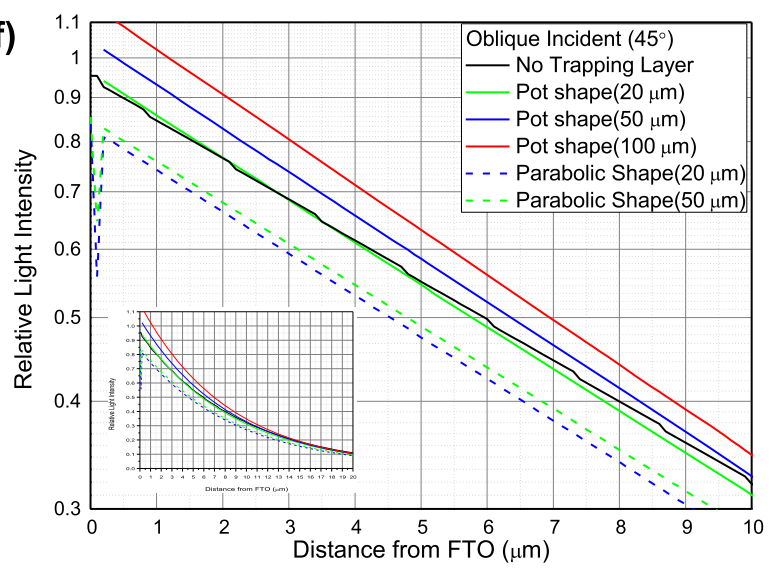

Fig. 3 a Light distribution in the light-trapping layer, substrate, and photoanode by light at normal incidence according to the light-trapping layer shape, i.e., (left) pot shape and (right) parabolic lens shape, with dimensions as given in Fig. 2. The light intensity distribution within the photoanode b without the light-trapping layer, $\mathbf{c}$ with a pot-shaped light-trapping layer, and $\mathbf{d}$ with a parabolic lens shape for (up) normal and (down) oblique incidence at $45^{\circ}$. The light distribution profile within the photoanode according to the distance from the interface between the photoanode and fluorine-doped tin oxide (FTO) film according to the light-trapping layer shape e for light at normal incidence and $\mathbf{f}$ oblique light at $45^{\circ}$ incidence.

resistance, decreased as the angle of incidence increased. For the same projection area, the resulting larger cell area provides higher efficiency.

Similar tendencies were observed for the $11.5 \mu \mathrm{m}$-thick photoanode, as shown in Fig. 4c, d). However, in this case, the effects of the incident angle was limited by higher recombination in the photoanode owing to its thickness, as shown in Fig. 4f. Thus, to enhance the energy conversion efficiency via light trapping, both the light distribution and recombination in the photoanode should be considered.
Taken together, these results provide insight into the design of more efficient DSSCs. The light-trapping layer can be modified to enhance the effective light absorption coefficient, i.e., charge-generation rate. To collect the generated charges more effectively, a large cell area with respect to the photoanode projection under illumination is a good solution. Further enhancement can be obtained through modification and optimization of the cell structure and its materials. For example, utilization of a very thin photoanode with a high light absorption dye ${ }^{29,30}$, such as an organic dye instead of one containing 

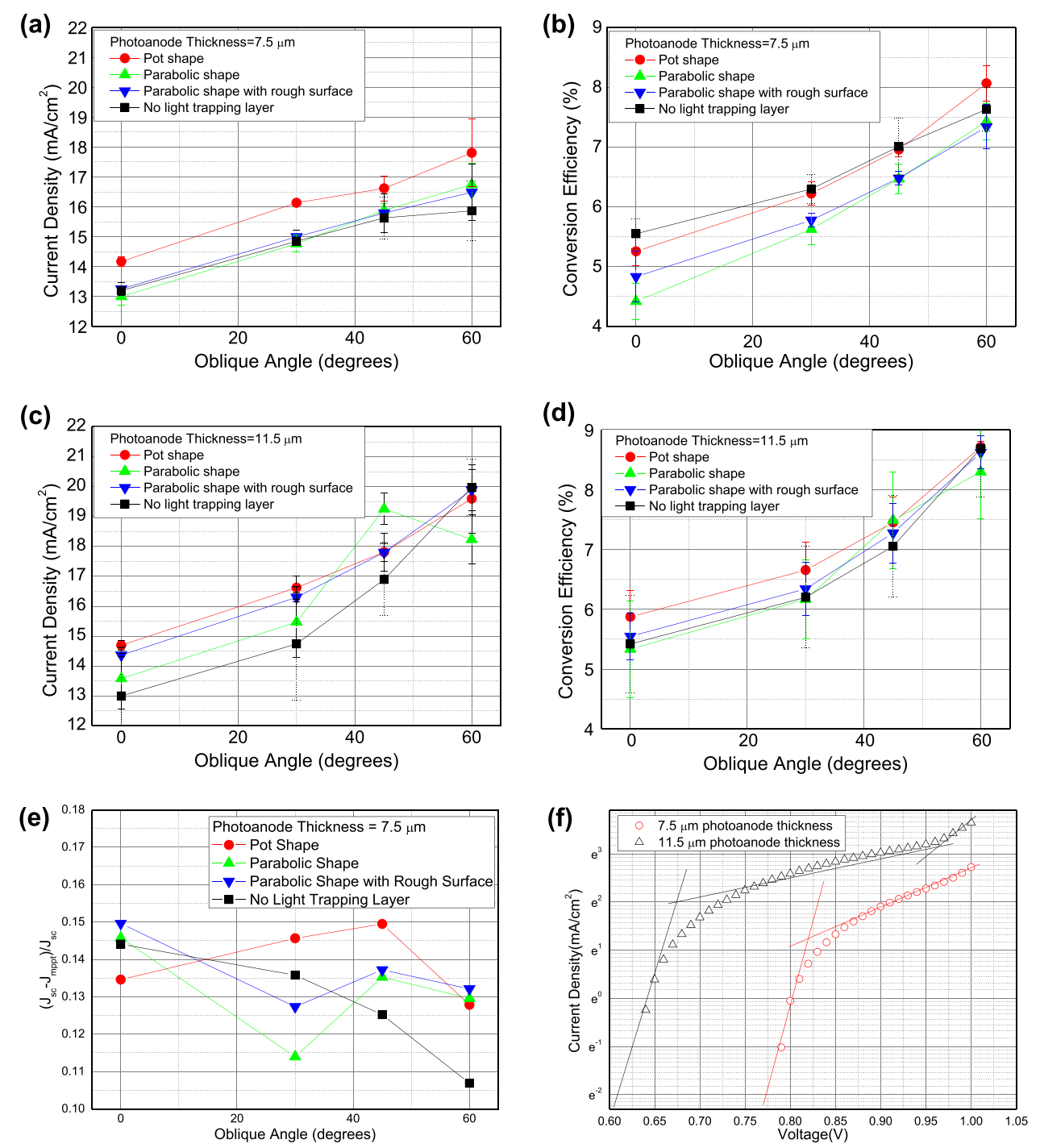

Fig. 4 a Short-circuit current density (Jsc) and $\mathbf{b}$ energy conversion efficiency of DSSCs under 1 sun AM1.5 using a $7.5 \mu$ m-thick photoanode as a function of the light incident angle and the shape of the light-trapping layer. $\mathbf{b} \mathrm{JSC}$ and $\mathbf{d}$ the energy conversion efficiency of DSSCs fabricated with a $11.5 \mu \mathrm{m}$-thick photoanode according to light-incident angle and the presence of a light-trapping layer. $\mathbf{e}$ The difference between Jsc and current density at the maximum power point according to the light-incident angle and the light-trapping layer. $\mathbf{f}$ The relationship between the logarithm of the current density and applied voltage over the open-circuit voltage.

ruthenium $(\mathrm{Ru})$ used in this study, should improve DSSC efficiency with the use of a light-trapping layer and angled array. In addition, the light-trapping layer and light incident angle are important design features. For example, if we utilized a $7.5 \mu \mathrm{m}$-thick photoanode and a conventional module in which the cells were arranged in a planar array, an efficiency of $5.5 \%$ was obtained. However, if the same cells were arranged at an angle of $60^{\circ}$, i.e., an angled array, the efficiency increased to $7.5 \%$. Given this arrangement, adding the photoanode light-trapping layer resulted in a maximum efficiency of $8.1 \%$. Using this submodule configuration and then increasing the photoanode thickness to $11.5 \mu \mathrm{m}$ resulted in an efficiency of $8.5 \%$, compared with $5.5 \%$ efficiency for individual cells. The results of the aforementioned adjustments demonstrate the importance of optimizing the materials used and the configurations of the light-trapping layer and cell structures. As such, further improvements in efficiency are expected for the proposed DSSC design.

Parallel arrays of four cells were fabricated as 3Darrayed submodules in which the arrays were flat or angled at $30^{\circ}, 45^{\circ}$, or $60^{\circ}$, as shown in Fig. 5a. The angled array of each cell induced an oblique incidence condition. Current density and energy conversion efficiency measurements were conducted on these DSSC submodules, with and without a light-trapping layer and with variation 
(a)

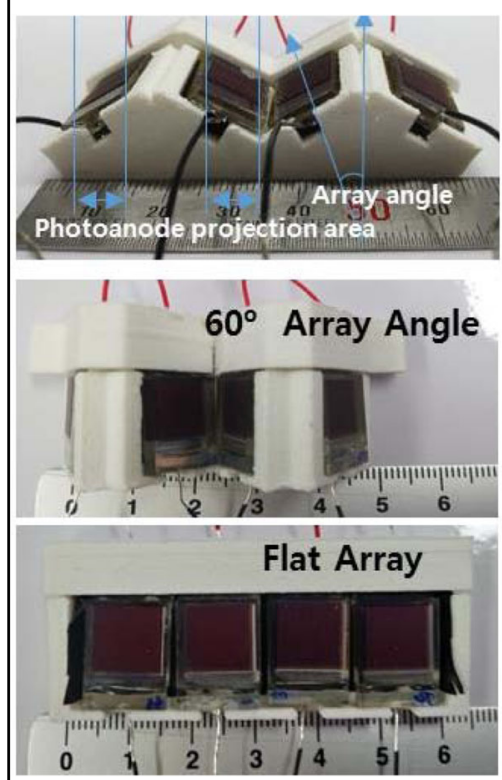

(b)

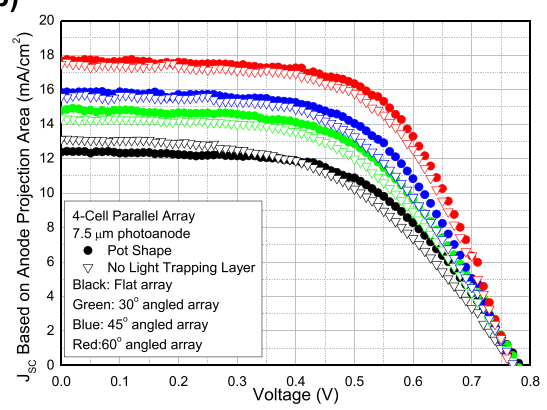

(d)

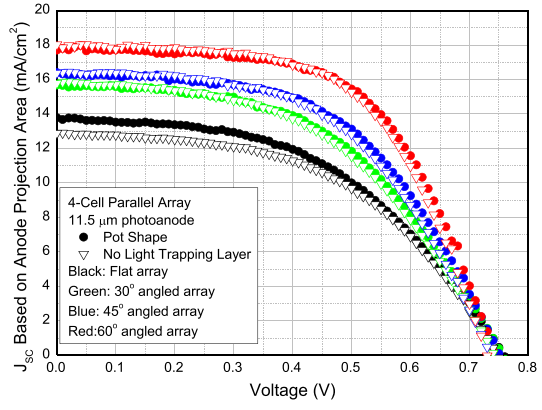

(c)

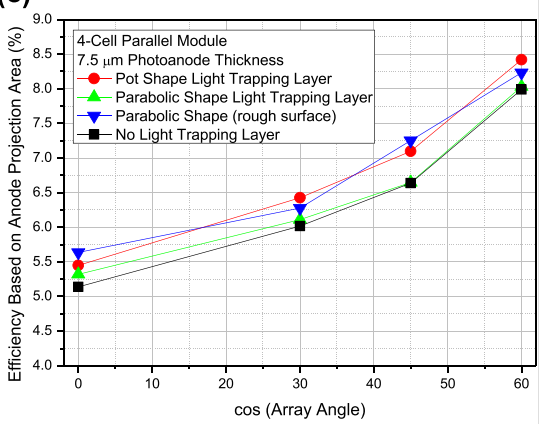

(e)

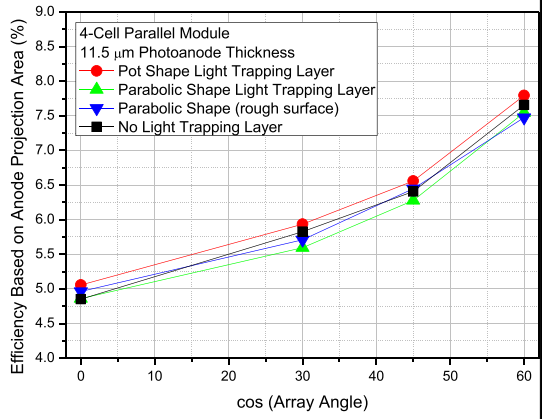

Fig. 5 a Photographs of three-dimensional (3D)-arrayed submodules consisting of four DSSCs with different array angles. The top picture indicates the definition of an array angle and the photoanode projection area used for current density and efficiency measurements. $\mathbf{b}$ The current density-voltage relationship of the DSSC submodule of cells with and without a light-trapping layer with different array angles and $\mathbf{c}$ their energy conversion efficiency for a photoanode thickness of $\mathbf{d} 7.5 \mu \mathrm{m}$ and $\mathbf{e} 11.5 \mu \mathrm{m}$. The current density-voltage relationship and efficiency were measured under 1 sun AM1.5 illumination at normal incidence with respect to the module surface.

in the photoanode thickness $(7.5$ and $11.5 \mu \mathrm{m})$, under 1 sun AM1.5 illumination at normal incidence with respect to the module surface (Fig. 5). To fix each cell in an angled array configuration and to prevent light from passing through the edges or sides of the cells, we used large frames to cover these parts; notably, proper module fabrication can eliminate or minimize this auxiliary area. In these submodules, the increased array angle induced a higher projection area-based efficiency, as shown in Fig. $5 \mathrm{~b}-\mathrm{e}$, regardless of the photoanode thickness. An efficiency of $8.5 \%$ was achieved by adding a $11.5 \mu \mathrm{m}$ lighttrapping layer and an array configuration in which the submodule was angled at $60^{\circ}$, compared with $5.5 \%$ efficiency obtained when the submodule was assembled in the conventional way as a flat array (Fig. 5a).

The improvements in DSSC efficiency were not limited to the energy conversion efficiency assuming a fixed installation area, as shown in Fig. 5. Experimental simulations of this module under 1-day operation and changing lighting conditions, i.e., in which the angle of incidence changed over time with respect to the submodule, showed that the angled array and the introduction of the light-trapping layer greatly increased electricity production in the fixed installation area, as shown in Fig. 6. Thus, the hidden possibilities of applying DSSCs for urban environment applications are promising. In addition, the results from this study provide new research directions for next-generation PVs via further improvements in DSSCs at the cell and submodule design levels. We believe these new directions include the development of thin photoanodes with higher absorption dyes, i.e., organic dyes, and optimization of the light-trapping design and cell structure to further improve DSSC energy conversion efficiency.

\section{Conclusion}

There has been great interest in developing the means to maximize electricity under low-intensity light illumination for PVs applications in urban environments, in which the projection area is limited or fixed. The results of this study demonstrate the potential of DSSCs for this purpose by adapting light-trapping layers with increased absorption in the photoanode for omnidirectional light trapping and using a 3D angled array to maximize the photoanode projection area. Our DSSCs submodule design, consisting of four individual DSSCs, achieved 8.5\% efficiency, compared with the $5 \%$ efficiency exhibited by individual cells; this was an extraordinary performance, given that the cell design, materials, light-trapping layer, and 3D array configuration had yet to be fully optimized. Our results suggest rethinking 

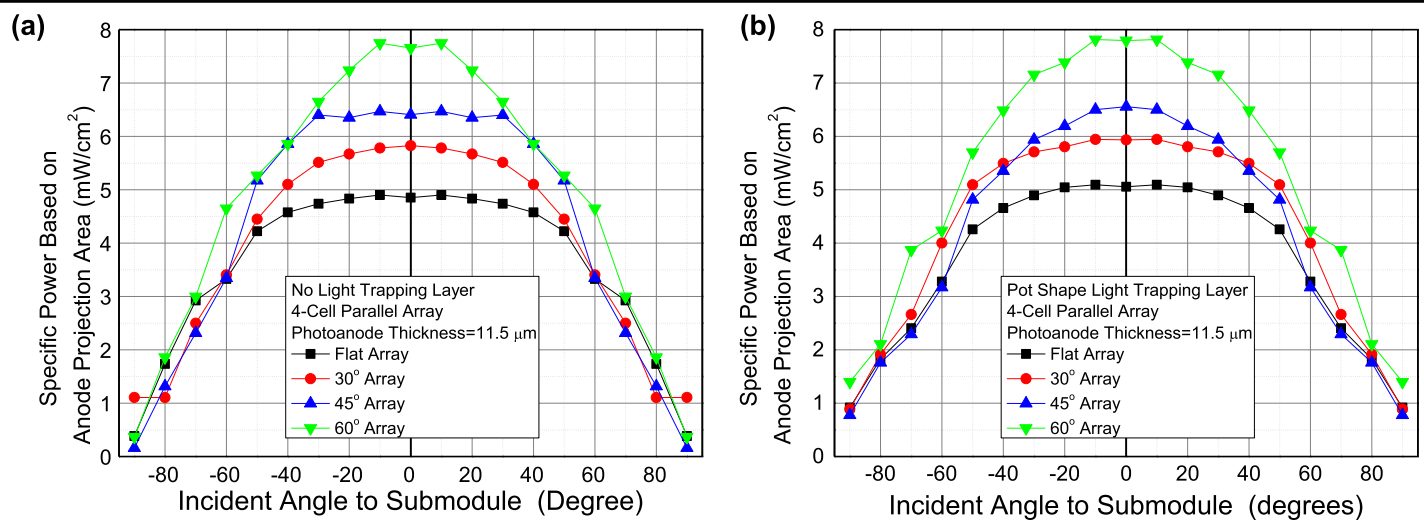

Fig. 6 Specific power output from submodules consisting of four DSSCs of a $11.5 \mu \mathrm{m}$-thick photoanode with different array angles as a function of incident angle to the submodules under 1 sun AM1.5 illumination, for submodules consisting of DSSC cells a without the light-trapping layer and b with a pot-shaped light-trapping layer.

the flat panel design inherited from conventional solar cells constructed using Si-based PVs modules. Our proposed DSSCs submodule design shows the feasibility of using DSSCs-based PVs in urban environments requiring solar cell panel installation in fixed-direction constrained areas.

\section{Acknowledgements}

This research was supported by a primary research program of the Korea Electrotechnology Research Institute (KERI) through the National Research Council of Science and Technology (NIST), funded by the Ministry of Science, ICT, and Future Planning (MSIP) (grant no. 19-12-N0101-28).

\section{Conflict of interest}

The authors declare that they have no conflict of interest.

\section{Publisher's note}

Springer Nature remains neutral with regard to jurisdictional claims in published maps and institutional affiliations.

Supplementary information is available for this paper at https://doi.org/ 10.1038/s41427-020-0196-9.

Received: 6 September 2019 Revised: 26 October 2019 Accepted: 28 October 2019.

Published online: 31 January 2020

\section{References}

1. Martinot, E., Cabraal, A. \& Mathur, S. World Bank/GEF solar home system projects: experiences and lessons learned 1993-2000. Renew. Sustain. Energy Rev. 5, 39-57 (2001).

2. Parida, B., Iniyan, S. \& Goic, R. A review of solar photovoltaic technologies. Renew. Sustain. Energy Rev. 15, 1625-1636 (2011).

3. Hamid, S. A., Othaman, M. Y., Sopian, K. \& Zaidi, S. H. An overview of photovoltaic thermal combination (PV/T combi) technology. Renew. Sustain. Energy Rev. 38, 212-222 (2014).

4. Wang, C. et al. Cost-effective anthryl dyes for dye-sensitized cells under one sun and dim light. J. Phys. Chem. C. 119, 24282-24289 (2015).

5. Devabhaktuni, V. et al. Solar energy: trends and enabling technologies. Renew. Sustain. Energy Rev. 19, 555-564 (2013).

6. Selvaraj, P. et al. Enhancing the efficiency of transparent dye-sensitized solar cells using concentrated light. Sol. Energy Mater. Sol. Cells 175, 29-34 (2018).

7. Yang, $\mathrm{N}$. in The preparation of nano composites and their applications in solar energy conversion 4, 81-91 (Springer-Verlag, Heidelberg, Berlin, Germany, 2017).
8. Hussain, F. Design development and performance evaluation of photovoltaic/ thermal (PV/T) air base solar collector. Renew. Sustain. Energy Rev. 25, 431-441 (2013).

9. Hyder, F., Sudhakar, K. \& Mamat, R. Solar PV tree design: a review. Renew. Sustain. Energy Rev. 82, 1079-1096 (2018).

10. Hyder, F., Baredar, P., Sudhakar, K. \& Mamat, R. Performance and land footprint analysis of a solar photovoltaic tree. J. Clean. Prod. 187, 432-448 (2018).

11. McMillon-Brown et al. Light-trapping in polymer solar cells by processing with nanostructured diatomaceous earth. Org. Electron. 51, 422-427 (2017).

12. Knott, A., Makarovskiy, O., O'Shea, J., Wu, Y. \& Tuck, C. Scanning photocurrent microscopy of 3D printed light trapping structures in dye-sensitized solar cells. Sol. Energy Mater. Sol. Cells 180, 103-109 (2018).

13. Reale, A. et al. Estimation of energy production of dye-sensitized solar cell modules for building-integrated photovoltaic applications. Energy Technol. 2, 531-541 (2014)

14. Cojocaru, L. et al. Determination of unique power conversion efficiency of solar cell showing hysteresis in the I-V curve under various light intensities. Sci. Rep. 7, 11790 (2017)

15. Chen, C. et al. Perovskite photovoltaics for dim-light applications. Adv. Funct. Mater. 25, 7064-7070 (2015).

16. Carlson, D. E. \& Wronski, C. R. Amorphous silicon solar cell. Appl. Phys. Lett. 28, 671-673 (1976)

17. Ni, M., Leung, M. K. H., Leung, D. Y. C. \& Sumathy, K. An analytical study of porosity effect on dye-snesitized solar cell performance. Sol. Energy Mater. Sol. Cells 90, 1331-1344 (2006).

18. Chen, C., Wu, S., Wu, C., Chen, J. \& Ho, K. A Ruthenium complex with superhigh light-harvesting capacity for dye-sensitized solar cells. Angew. Chem. 118, 5954-5957 (2006).

19. Yun, M. J., Sim, Y. H., Cha, S. I., Seo, S. H. \& Lee, D. Y. 3-Dimensional dye sensitized solar cell sub-module with oblique angled cell array for enhanced power and energy density output utilizing non-linear relation in cosine law of light incident angle. Sol. Energy 177, 355-363 (2019).

20. Szmytkowski, J. The influence of the thickness, recombination and space charge on the loss of photocurrent in organic semiconductors: an analytical model. J. Phys. D Appl. Phys. 40, 3352-3357 (2007).

21. Baglio, V., Girolamo, M., Antonucci, V. \& Arico, A. S. Influence of $\mathrm{TiO}_{2}$ film thickness on the electrochemical behaviour of dye-sensitized solar cells. Int. J. Electrochem. Sci. 6, 3375-3384 (2011).

22. Fang, C. et al. Antireflective paraboloidal microlens film for boosting power conversion efficiency of solar cells. ACS Appl. Mater. Interfaces 10, 21950-21956 (2018).

23. Kim, D. H., Dudem, B., Jung, J. W. \& Yu, J. S. Boosting light harvesting in perovskite solar cells by biomimetic inverted hemispherical architectured polymer layer with high haze factor as an antireflective layer. ACS Appl. Mater. Interfaces 10, 13113-13123 (2018).

24. Senthil, R. \& Yuvaraj, S. A comprehensive review on bioinspired solar photovoltaic cells. Int. J. Energy Res. 43, 1-14 (2018). 
25. Lin, T. Y., Huang, Y. Y., Tsao, C. W., Pan, J. W. \& Wang, C. M. Efficiency evaluation of a hybrid miniaturized concentrated photovoltaic for harvesting direct/diffused solar light. J. Opt. 21, 035901 (2019).

26. Escalante, $\mathrm{R}$. et al. Influence of $\mathrm{TiO}_{2}$ film thickness on the performance of dyesensitized solar cells: relation between optimum film thickness and electron diffusion length. Energy Environ. Focus. 2, 280-286 (2013).

27. Tan, G. L., Lemon, M. F., Jones, D. J. \& French, R. H. Optical properties and London dispersion interaction of amorphous and crystalline $\mathrm{SiO}_{2}$ determined by vacuum ultraviolet spectroscopy and spectroscopic ellipsometry. Phys. Rev. B. 72, 205117 (2005)
28. Cai, Z., Qiu, W., Shao, G. \& Wang, W. A new fabrication method for all-PDMS waveguides. Sens. Actuators A 204, 44-47 (2013).

29. Howie, W. H., Claeyssens, F., Miura, H. \& Peter, L. M. Characterization of solidstate dye-sensitized solar cells utilizing high absorption coefficient metal-free organic dyes. J. Am. Chem. Soc. 130, 1367-1375 (2008).

30. Wu, Y. et al. High-conversion-efficiency organic dye-sensitized solar cells: molecular engineering on D-A-T-A featured organic indoline dyes. Energy Environ. Sci. 5, 8261-8272 (2012). 